\title{
PREDICTIVE CONTROL APPROACH FOR PERMANENT MAGNET SYNCHRONOUS MOTOR DRIVE
}

\author{
Arthur G. Bartsch, Gabriel H. Negri, Camila R. Scalabrin, Mariana S. M. Cavalca, Ademir Nied, \\ José de Oliveira \\ Santa Catarina State University, Joinville - SC, Brazil \\ e-mail: \{arthurbartsch, negri.gabriel, c.scalabrin\}@gmail.com, \{mariana.cavalca, ademir.nied, jose.oliveira\}@udesc.br
}

\begin{abstract}
This paper presents a predictive control approach for speed control of a permanent magnet synchronous motor with trapezoidal back-electromotive force drive. The prediction model was numerically identified and considers existent transport delays in the drive. The proposed technique operates with sixstep and pulse-width modulations, which are normally used in proportional-integrative control structures. A computational cost analysis was also done. Results show improvements in speed performance, comparing to tested proportional-integral control.
\end{abstract}

Keywords - Permanent Magnet Synchronous Motor, Predictive Control, Six-step Modulation, Transport Delay.

\section{INTRODUCTION}

Permanent magnet synchronous motors (PMSMs) are known for having high efficiency, good speed dynamics and long life cycle [1]. There are two main types of PMSMs: those with sinusoidal back-electromotive force (back-EMF) and those with trapezoidal back-EMF [2], [3]. The last type is also known as "brushless direct current" motor (BLDCM), although it is an alternating current motor [3], [4].

Both types of motors have similar characteristics. However, for applications that can support some torque ripple, the PMSM with trapezoidal back-EMF is normally used, since this motor has a smaller acquisition cost in relation to sinusoidal back-EMF motor [1], [3]. PMSMs with trapezoidal back-EMF are applied in refrigerators, respirators and, even, electrical vehicles [5]-[7].

PMSMs normally do not operate in open loop, due to there is not current limitation. Thus, drive efficiency and security are prejudiced. For the sinusoidal PMSM, field oriented control or direct torque control are employed, to deal with sinusoidal signals [3], [7]-[10]. For BLDCM, it is possible to use these techniques, but reference frame model is not completely valid, since neutral voltage is not null [3], [4], [11]. Discontinuous six-step modulation is often used to drive BLDCM. Such modulation reduces losses in inverter switches, since one phase is disconnected at each modulation step [3], [12], [13]. Pulse-width modulation (PMW) is combined with six-step for speed control. To determine duty cycle for PWM, usually, a proportional-integrative (PI) controller is used [3].

Model-based predictive control (MPC) is a family of algorithms that minimizes a cost function, based on an explicit

Manuscript received 20/08/2015. First revision 21/10/2015; second revision $15 / 12 / 2015$. Accepted for publication $15 / 12 / 2015$, by recommendation of the Regular Section Editor Cassiano Rech. mathematical model of a plant. Such control techniques first appeared in petrochemical industry patents, in the decade of 1970. Since that epoch, many predictive control approaches were developed, as generalized predictive control (GPC) [14], dynamic matrix control (DMC) [14] and state space modelbased predictive control (SSMPC) [13]-[16] among others, being utilized in a variety of applications [14].

In industrial electronics, two research lines using SSMPC are highlighted: the continuous control set (CCS) [13], [17] and the finite control set (FCS) approaches [18], [19]. The first considers control action is a real number. In this case, the switching effect is neglected and an average model is used for prediction. The second considers a finite number of control actions, based on possible combinations of active switches in a converter. This way, a nonlinear model is used for prediction.

Predictive control approaches have various advantages in relation to controllers designed using frequency approaches. Among these advantages are: capacity to handle easily with multiple input multiple output (MIMO) plant, capacity to deal with existing transport delays in the plant, to treat process constraints in the control project. Predictive controllers design covers a cost function minimization, which allows intuitive tune. In particular, for motor drive systems, these controllers can minimize the spent energy, improving the drive efficiency.

This work proposes the use of a SSMPC approach, instead of a PI controller, in a PMSM drive, to improve drive dynamics. An identified model is used due to complexity of inverter-motor drive system model. The procedure used to perform this identification is detailed in [13]. However, there are some additional contributions in this paper. First, in current work, the identified prediction model is used to start the motor. Second, a first order model is presented, which reduces control computational cost. Third, delays existing on the drive are treated. Fourth, the integral action was done using a virtual reference state instead the use of incremental action [20]. Fifth, a comparative study between the predictive controller and the PI controller is done. Finally, an experimental computational cost analysis is presented.

Works using different simplified models, as average models, operation stages models, DC motor models can be found in [4], [6], [21]. All of these models are used to make the control design easier.

In Section II, MPC theory, in this work scope, is presented. In Section III, drive structure, six-step modulation and numerical model identification are discussed. In Section IV, the methodology used to obtain the experimental results is presented. Section V shows the experimental results with discussion about them. Concluding remarks are presented in Section VI. 


\section{PREDICTIVE CONTROL THEORY}

In this paper, PMSM speed is controlled using state space model-based predictive control (SSMPC). Generally, predictive controllers, such as SSMPC, perform a cost function minimization, based on a prediction model.

The chosen cost function indicates priorities in the optimization process. Considering a single input single output (SISO) system, a quadratic cost function $q(k)$ is defined as

$$
q(k)=\left(Y_{r}-Y\right)^{\mathrm{T}}\left(Y_{r}-Y\right)+\rho U^{\mathrm{T}} U
$$

where $Y_{r}$ is a vector with future references $y_{r}\left(k+\xi_{y}\right), \xi_{y} \in$ $\left[1, h_{p}\right], Y$ is a vector of predicted outputs $y\left(k+\xi_{y}\right), \rho$ is a weighing coefficient and $U$ is a vector that contains future calculated control actions $u\left(k+\xi_{u}\right), \xi_{u} \in\left[0, h_{c}-1\right]$. Outputs are predicted in a discrete time horizon, called prediction horizon $h_{p}$. Future control actions are also calculated in a discrete time horizon, known as control horizon $h_{c}\left(h_{c} \leq h_{p}\right)$. This way,

$$
\begin{gathered}
Y_{r}=\left[\begin{array}{c}
y_{r}(k+1) \\
y_{r}(k+2) \\
\vdots \\
y_{r}\left(k+h_{p}\right)
\end{array}\right], Y=\left[\begin{array}{c}
y(k+1 \mid k) \\
y(k+2 \mid k) \\
\vdots \\
y\left(k+h_{p} \mid k\right)
\end{array}\right] \\
U=\left[\begin{array}{c}
u(k \mid k) \\
u(k+1 \mid k) \\
\vdots \\
u\left(k+h_{c}-1 \mid k\right)
\end{array}\right] .
\end{gathered}
$$

The notation $y\left(k+\xi_{y} \mid k\right)$ represents the output prediction for the $\xi_{y}$-th future sampling period, given the information acquired in the sampling instant $k$.

From a discrete linear time-invariant state space SISO model, it is possible to perform predictions using a linear SSMPC approach. The state-space model is given by:

$$
\begin{aligned}
X(k+1) & =\mathbf{A} X(k)+\mathbf{B} u(k) \\
y(k) & =\mathbf{C} X(k)
\end{aligned}
$$

where $\mathbf{A}$ is the state dynamic matrix, $B$ is the input system vector, $\mathbf{C}$ is the output system matrix and $X$ is the state vector, with order $n$, described as

$$
X(k)=\left[\begin{array}{llll}
x_{1}(k) & x_{2}(k) & \ldots & x_{n}(k)
\end{array}\right]^{\mathrm{T}} .
$$

In addition, one can consider that [14]

$$
Y=\mathbf{G} U+\Phi X
$$

where

$$
\begin{gathered}
\mathbf{G}=\left[\begin{array}{cccc}
\mathbf{C B} & 0 & \ldots & 0 \\
\mathbf{C A B} & \mathbf{C B} & \ldots & 0 \\
\vdots & \vdots & \ddots & \vdots \\
\mathbf{C A}^{h_{p}} \mathbf{B} & \mathbf{C A}^{h_{p}-1} \mathbf{B} & \ldots & \mathbf{C A}^{h_{p}-h_{c}} \mathbf{B}
\end{array}\right], \\
\Phi=\left[\begin{array}{llll}
\mathbf{C A} & \mathbf{C A}^{2} & \ldots & \mathbf{C A}^{h_{p}}
\end{array}\right]^{\mathrm{T}} .
\end{gathered}
$$

An analytic solution for the minimization problem of (1) subject to (6) is obtainable. Thus, the optimal control sequence $U^{*}$ is given by

$$
U^{*}=\mathbf{K}_{\mathbf{c}}\left(Y_{r}-\Phi X\right)
$$

where $\mathbf{K}_{c}$ is the optimal gain, calculated with

$$
\mathbf{K}_{\mathbf{c}}=\left(\mathbf{G}^{\mathrm{T}} \mathbf{G}+\rho I\right)^{-1} \mathbf{G}^{\mathrm{T}}
$$

where $I$ is the identity matrix, with order $h_{c}$. Matrices $\mathbf{G}, \Phi$ and $\mathbf{K}_{\mathbf{c}}$ are calculated off-line in this control approach. This way, in an embedded application, it is not necessary to invert a matrix on-line in the digital controller.

Only the first term of (9) is applied to the plant, which characterizes the receding horizon strategy.

To apply the control strategy, the off-line steps are:

1. To define a motor model, with matrices $\mathbf{A}, B$ and $\mathbf{C}$.

2. To calculate matrices $\mathbf{G}, \Phi$ and $\mathbf{K}_{\mathbf{c}}$.

3. To create a internal future speed reference function, if possible.

The on-line steps of this predictive control technique are:

1. To generate the reference vector $Y_{r}$. If the future references are unknown, it is possible to maintain all future reference values constant.

2. To calculate the term $\Phi X$, named as free response.

3. To perform the subtraction $Y_{r}-\Phi X$.

4. To calculate $u(k)$, choosing the first term of (9).

5 . To saturate $u(k)$, if necessary.

6. To send duty cycle to embedded device PWM module.

7. To wait the next sampling time and return to step 1.

\section{DRIVE MODELING}

MPC requires an explicit mathematical model of the plant. In this case, the plant is composed of the PMSM and the inverter, considering the chosen modulation strategy. Thus, it is necessary to define each part of the drive system in order to obtain the prediction model.

\section{A. Motor Information}

PMSMs with trapezoidal back-EMF do not have a field winding in the rotor unlike a conventional synchronous motor. Instead, these motors have permanent magnets for excitation. This excitation inducts a back-EMF in stator, which actuates as a trapezoidal voltage source.

The trapezoidal form of the back-EMF causes a non-null voltage in the neutral point of the stator. Thus, simplifications normally applied to sinusoidal PMSMs are not valid for BLDCMs [4].

\section{B. Drive System Information}

The most common mode to drive BLDCMs is performing an electronic commutation. This can be done by using discontinuous six-step modulation. Its effect is similar of a conventional DC motor commutation [3], [4], [11]. This way, at each 60 electrical degrees, one phase of the motor is opened, considering back-EMF trapezoidal form. Each phase conducts current for 240 electrical degrees per rotor electrical cycle. 


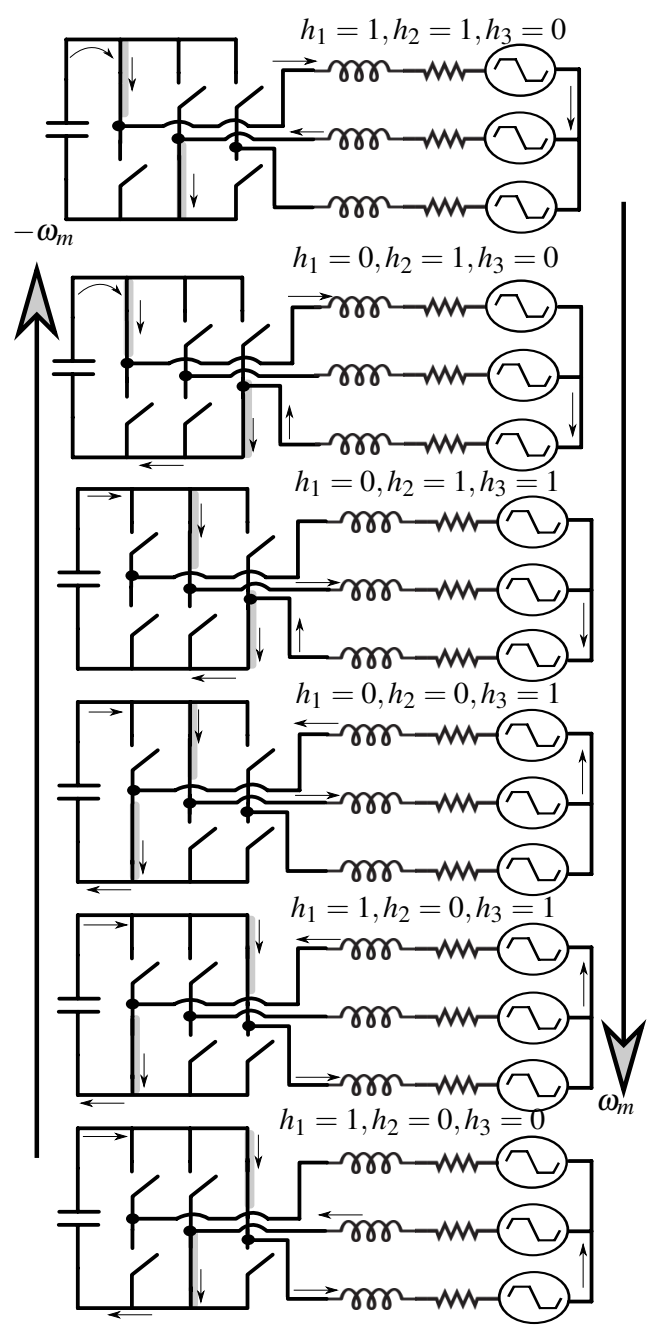

Fig. 1. Operation stages of six-step commutation $\left(h_{1}, h_{2}\right.$ and $h_{3}$ are the logical levels of the Hall effect sensors).

Position information is obtained with three Hall effect sensors $h_{1}, h_{2}$ and $h_{3}$, internally located in the motor. They are exactly placed in the positions of 0,120 and -120 electrical degrees, in relation to phase $a$, respectively. These sensors have low cost, since they only operate in cut-off and saturation regions, informing high logical level for a North field and low level for a South field. Figure 1 presents six-step commutation logic.

In the conductive phases, PWM is applied, allowing speed control, as an average voltage applied in each phase. Due to this, switching frequency should be greater than the sampling frequency, used in the control, to allow average voltage effect.

\section{System Analytical Modeling}

For this modeling, each switch $\delta$ of inverter is treated as a resistance, given by:

$$
r_{\delta}= \begin{cases}0 & \text { if active } \\ \infty & \text { if inactive }\end{cases}
$$

Furthermore, it is considered that the motor has symmetrical impedance distribution per phase and it does not have any magnetic non-linearities, as saturation, hysteresis or eddy currents. Drive equivalent circuit is shown in Figure 2.

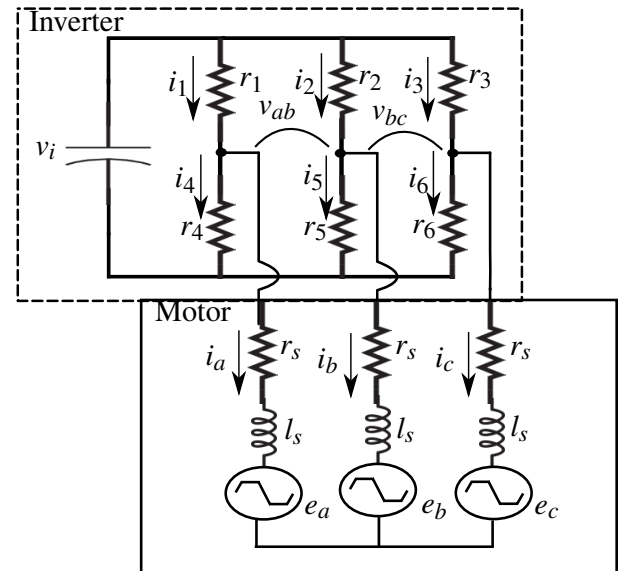

Fig. 2. Equivalent circuit for the drive system, used in modeling.

Using Euler's backward discretization, the discrete electrical model of motor-inverter system is given by

$$
\begin{aligned}
{\left[\begin{array}{c}
i_{a}(k+1) \\
i_{b}(k+1) \\
i_{c}(k+1)
\end{array}\right]=} & {\left[\begin{array}{ccc}
1-2 \zeta_{a} & \zeta_{b} & \zeta_{c} \\
\zeta_{a} & 1-2 \zeta_{b} & \zeta_{c} \\
\zeta_{a} & \zeta_{b} & 1-2 \zeta_{c}
\end{array}\right]\left[\begin{array}{c}
i_{a}(k) \\
i_{b}(k) \\
i_{c}(k)
\end{array}\right] } \\
+ & {\left[\begin{array}{rrrr}
\zeta_{x} & -2 \zeta_{u} & \zeta_{u} & \zeta_{u} \\
\zeta_{y} & \zeta_{u} & -2 \zeta_{u} & \zeta_{u} \\
\zeta_{z} & \zeta_{u} & \zeta_{u} & -2 \zeta_{u}
\end{array}\right]\left[\begin{array}{c}
v_{i}(k) \\
e_{a}(k) \\
e_{b}(k) \\
e_{c}(k)
\end{array}\right] }
\end{aligned}
$$

where

$$
\begin{aligned}
& \zeta_{u}=\frac{t_{s}}{l_{s}}, \zeta_{a}=\frac{r_{x}+r_{s}}{l_{s}} t_{s}, \zeta_{b}=\frac{r_{y}+r_{s}}{l_{s}} t_{s}, \zeta_{c}=\frac{r_{z}+r_{s}}{l_{s}} t_{s} \\
& \zeta_{x}=\frac{t_{s}}{l_{s}}\left(\frac{2 r_{x}}{r_{1}}-\frac{r_{y}}{r_{2}}-\frac{r_{z}}{r_{3}}\right) \\
& \zeta_{y}=\frac{t_{s}}{l_{s}}\left(-\frac{r_{x}}{r_{1}}+\frac{2 r_{y}}{r_{2}}-\frac{r_{z}}{r_{3}}\right) \\
& \zeta_{z}=\frac{t_{s}}{l_{s}}\left(-\frac{r_{x}}{r_{1}}-\frac{r_{y}}{r_{2}}+\frac{2 r_{z}}{r_{3}}\right) \\
& r_{x}=\frac{r_{1} r_{4}}{r_{1}+r_{4}}, r_{y}=\frac{r_{2} r_{5}}{r_{2}+r_{5}}, r_{z}=\frac{r_{3} r_{6}}{r_{3}+r_{6}} \\
& e_{a}(k)=k_{e} \omega_{m}(k) \operatorname{tra}\left(\theta_{e}(k)\right) \\
& e_{b}(k)=k_{e} \omega_{m}(k) \operatorname{tra}\left(\theta_{e}(k)-\frac{2 \pi}{3}\right) \\
& e_{c}(k)=k_{e} \omega_{m}(k) \operatorname{tra}\left(\theta_{e}(k)+\frac{2 \pi}{3}\right)
\end{aligned}
$$

and $l_{s}$ is the motor inductance, $r_{s}$ is the motor resistance, $t_{s}$ is the control sampling time, $r_{1}-r_{6}$ are the equivalent switches resistances, $k_{e}$ is the electromotive force constant, $\theta_{e}(k)$ is the electrical position and $\operatorname{tra}(\theta(k))$ is the normalized trapezoidal function. Mechanical dynamics can be expressed as

$$
\omega_{m}(k+1)=\left(1-\frac{b_{m} t_{s}}{j_{m}}\right) \omega_{m}(k)+\frac{\tau_{e}(k)-\tau_{m}}{j_{m}} t_{s}
$$

where $\omega_{m}(k)$ is the mechanical speed, $b_{m}$ is the friction coefficient, $j_{m}$ is the inertia, $\tau_{m}$ is the load torque and $\tau_{e}(k)$ 
is the electrical torque, given by

$$
\tau_{e}(k)=k_{t}\left[i_{a} \operatorname{tra}\left(\theta_{e}\right)+i_{b} \operatorname{tra}\left(\theta_{e}-\frac{2 \pi}{3}\right)+i_{c} \operatorname{tra}\left(\theta_{e}+\frac{2 \pi}{3}\right)\right]
$$

where $k_{t}$ is torque constant. In (22), $i_{a}, i_{b}, i_{c}$ and $\theta_{e}$ are discrete-time varying functions.

Finally, the relation between mechanical and electrical variables is given by

$$
\begin{gathered}
\theta_{e}(k)=\frac{n_{p}}{2} \theta_{m}(k) \\
\theta_{m}(k)=\sum_{l=0}^{k-1} \omega_{m}(l) t_{S}
\end{gathered}
$$

where $n_{p}$ is number of poles.

\section{System Numerical Modeling}

As the analytical model is much complex for being a prediction model, an alternative method for modeling the drive was employed, using a numerical identification. The identified model describes speed behavior (output) as a function of duty cycle (input) [13]. As mechanical dynamics are represented by a first order equation, a first order model was chosen for identification. This way, it is necessary to obtain experimental data of speed behavior for this modeling technique. The speed response experimental data was obtained using an empirically tuned PI controller.

With input and output data, the least squares method was used to obtain the model parameters. In particular case, for a first order model, where $n_{d p}$ the number of data points [13],

$$
\left[\begin{array}{c}
y(1) \\
y(2) \\
\vdots \\
y\left(n_{d p}\right)
\end{array}\right]=\left[\begin{array}{cc}
y(0) & u(0) \\
y(1) & u(1) \\
\vdots & \vdots \\
y\left(n_{d p}-1\right) & u\left(n_{d p}-1\right)
\end{array}\right]\left[\begin{array}{l}
\gamma_{0} \\
\gamma_{1}
\end{array}\right]
$$

thus,

$$
Y=\mathbf{H} \Gamma
$$

and, this way, the regressors vector $\Gamma$ is given by

$$
\Gamma=\left(\mathbf{H}^{\mathrm{T}} \mathbf{H}\right)^{-1} \mathbf{H}^{\mathrm{T}} Y .
$$

With this procedure, an ARX (autoregressive with exogenous input) model is obtained, represented by

$$
y(k+1)=\gamma_{0} y(k)+\gamma_{1} u(k) .
$$

Considering $X(k)=x(k)=y(k)$, it is possible to rewrite the ARX model into a state space model. This way, $\mathbf{A}=\gamma_{0}, \mathbf{B}=\gamma_{1}$ and $\mathbf{C}=1$. With this:

$$
\begin{aligned}
x(k+1) & =\gamma_{0} x(k)+\gamma_{1} u(k) \\
y(k) & =x(k) .
\end{aligned}
$$

\section{E. Prediction Model}

Modulation, dead time, sampling process, control action calculation, switches rise and fall times are responsible for delays in the system [21]. Also, torque generation process

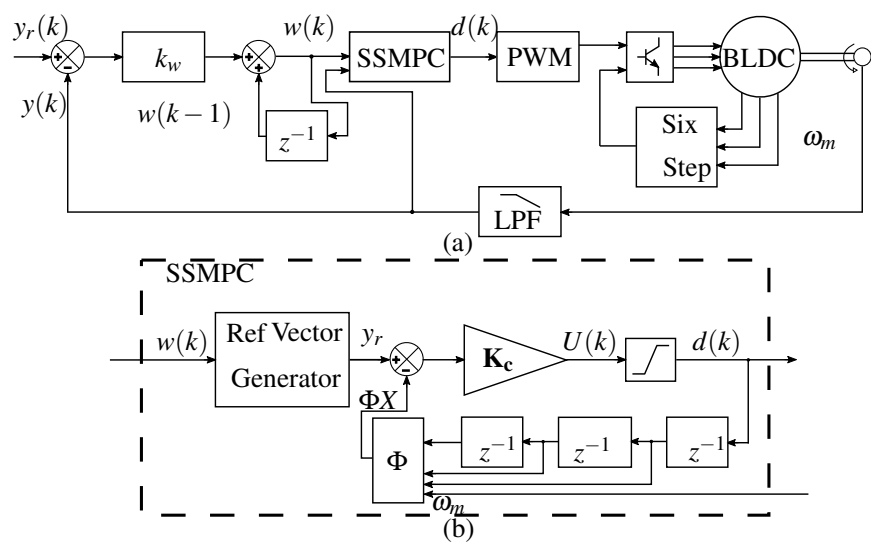

Fig. 3. (a)Proposed predictive control scheme to drive PMSM with Six-step. (b)Detailed SSMPC block.

includes a delay [18].This way, considering that

$$
\begin{aligned}
x(k+1) & =\gamma_{0} x(k)+\gamma_{1} u\left(k-n_{d}\right) \\
y(k) & =x(k)
\end{aligned}
$$

where $n_{d}$ is the number of delays in the process, it is possible to create additional states, for modeling such delays. Using

$$
\begin{gathered}
X_{a}(k)=\left[\begin{array}{cccc}
x(k) & u(k-1) & \ldots & u\left(k-n_{d}\right)
\end{array}\right]^{\mathrm{T}} \\
\mathbf{A}_{a}=\left[\begin{array}{ccccc}
\gamma_{0} & 0 & \ldots & 0 & \gamma_{1} \\
0 & 0 & \ldots & 0 & 0 \\
0 & 1 & \ldots & 0 & 0 \\
\vdots & \vdots & \ddots & \vdots & \vdots \\
0 & 0 & \ldots & 1 & 0
\end{array}\right] \\
\mathbf{B}_{a}=\left[\begin{array}{ccccc}
0 & 1 & \ldots & 0 & 0
\end{array}\right]^{\mathrm{T}} \\
\mathbf{C}_{a}=\left[\begin{array}{lllll}
1 & 0 & \ldots & 0 & 0
\end{array}\right]
\end{gathered}
$$

the augmented state-space model becomes

$$
\begin{aligned}
X_{a}(k+1) & =\mathbf{A}_{a} X_{a}(k)+\mathbf{B}_{a} u(k) \\
y(k) & =\mathbf{C}_{a} X_{a}(k) .
\end{aligned}
$$

Using (35), it is necessary that $h_{p}>n_{d}$ and $h_{c}>n_{d}$.

To include integral control action, an extra state was added to the model. This state is related to the integral of the tracking error and generates a new reference to the control (see Figure 3) [20]. This way:

$$
\mathbf{A}=\left[\begin{array}{cc}
\mathbf{A}_{a} & Z \\
-k_{w} \mathbf{C}_{a} & 1
\end{array}\right], \mathbf{B}=\left[\begin{array}{c}
\mathbf{B}_{a} \\
0
\end{array}\right], \mathbf{C}=\left[\begin{array}{ll}
\mathbf{C}_{a} & 0
\end{array}\right]
$$

where $Z$ is a zeros vector. The states vector becomes

$$
X(k)=\left[\begin{array}{ll}
X_{a}(k) & w(k)
\end{array}\right]^{\mathrm{T}}
$$

with $w$ defined as

$$
w(k)=w(k-1)+k_{w}\left[y_{r}(k)-y(k)\right] .
$$

The model presented in (36), (37) and (38) guarantees rejection of constant disturbances at the output and null steady state error for step references. 


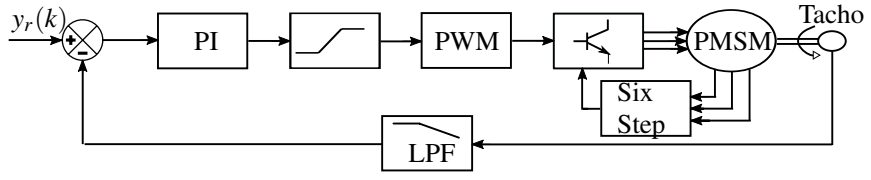

Fig. 4. Conventional PI control scheme for PMSM drive.

\section{METHODOLOGY}

In this paper, only experimental results are presented. These results were obtained using TWR-56F8400 development kit, from Freescale Semiconductor. This kit has a digital signal controller (DSC) with $100 \mathrm{MHz}$ of processing capability. The DSC is programmed in pure C language. A DC tacho-generator was used for speed measurement. A $24 \mathrm{~Hz}$ low-pass filter was used for noise reduction. All figures with results were generated in freeware GnuPlot, having 8000 points. Experimental data were obtained using ScopeCorder DL850E, from Yokogawa [22]. The steps of this work are:

- to obtain an identified numerical model for the drive;

- to compare the proposed control scheme with the traditional six-step, which uses a PI controller;

- to observe the influence of energy penalization in cost function presented in the Section III, showing how easy is to control the energy flux with predictive control;

- to evaluate the computational cost of proposed control.

To identify the model, following actions were taken:

1. driving the system with a empirical PI controller;

2. using (27) to get the model parameters $\gamma_{0}$ and $\gamma_{1}$;

3. validating the obtained model.

For the comparison with PI controller, the following procedure was used:

1. obtaining experimental data of various PI tunings;

2. acquiring the experimental data of proposed control;

3. calculating average quadratic tracking error, for dynamic performance comparison;

4. calculating root-mean-squared duty cycle, for energy performance comparison;

5. verifying other dynamics criteria.

The average quadratic tracking error $q_{e}$ is defined as

$$
q_{e}=\frac{1}{n_{d p}} \sum_{k=1}^{n_{d p}}\left[y(k)-y_{r}(k)\right]^{2} .
$$

The root-mean-squared duty cycle $q_{u}$ is defined as

$$
q_{e}=\sqrt{\frac{1}{n_{d p}} \sum_{k=1}^{n_{d p}}[u(k)]^{2}} .
$$

Figure 4 exhibits the PI control scheme. The PIs design was done as shown bellow:

- Equivalent continuous model from identified model was obtained (the model includes feedback filter response).
TABLE I

PI Tunings

\begin{tabular}{ccccc}
\hline Controller & $k_{i}^{\square}$ & $k_{p}^{\triangleright}$ & $\mathrm{PM}^{\dagger}$ & $f_{c}^{\dagger}$ \\
\hline PI 01 & 0.015 & $8.5 e^{-5}$ & $40^{\circ}$ & 6 \\
\hline PI 02 & 0.025 & $9.5 e^{-5}$ & $60^{\circ}$ & 3 \\
\hline PI 03 & 0.050 & $1.0 e^{-3}$ & $60^{\circ}$ & 19 \\
\hline PI 04 & 0.001 & $2.0 e^{-3}$ & $70^{\circ}$ & 40 \\
\hline PI 05 & 0.065 & $7.4 e^{-4}$ & $25^{\circ}$ & 11 \\
\hline PI 06 & 0.500 & $1.0 e^{-2}$ & $65^{\circ}$ & 80 \\
\hline PI 07 & 0.005 & $9.0 e^{-5}$ & $65^{\circ}$ & 3 \\
\hline PI 08 & 0.015 & $9.0 e^{-5}$ & $45^{\circ}$ & 6 \\
\hline PI 09 & 0.018 & $8.0 e^{-5}$ & $35^{\circ}$ & 7 \\
\hline PI 10 & 0.003 & $9.5 e^{-5}$ & $80^{\circ}$ & 2 \\
\hline
\end{tabular}

$\square$ Continuous integral gain.

${ }^{\triangle}$ Continuous proportional gain.

${ }^{\dagger}$ Projected values for open-loop system.

TABLE II

\begin{tabular}{|c|c|c|c|}
\hline \multicolumn{2}{|c|}{ Motor Model Parameters } & \multicolumn{2}{|c|}{ Control Parameters } \\
\hline$r_{s}$ & $0.5 \Omega$ & $h_{p}$ & $n_{d}+2$ \\
\hline$l_{s}$ & $0.436 \mathrm{mH}$ & $h_{c}$ & $n_{d}+2$ \\
\hline$n_{p}$ & 4 & $\rho_{1}$ & $1.5 \cdot 10^{4}$ \\
\hline$j_{m}$ & $1.6 \mu \mathrm{N} \mathrm{m} \mathrm{s}^{2}$ & $\rho_{2}$ & 750 \\
\hline$b_{m}$ & $26 \mu \mathrm{N} \mathrm{m} \mathrm{s}$ & $k_{w}$ & 0.1 \\
\hline$k_{e}$ & $0.01456 \mathrm{Vs}$ & $t_{s}$ & $1.0 \mathrm{~ms}$ \\
\hline$k_{t}$ & $0.01139 \mathrm{~N} \mathrm{~m} \mathrm{~A}^{-1}$ & $n_{d}$ & 3 \\
\hline \multicolumn{2}{|c|}{ Identified Model Parameters } & \multicolumn{2}{|c|}{ Inverter Parameters } \\
\hline$\gamma_{0}$ & 0.9768689 & $v_{i}$ & $24 \mathrm{~V}$ \\
\hline$\gamma_{1}$ & 11.419708 & $f_{s}$ & $16 \mathrm{kHz}$ \\
\hline \multicolumn{2}{|c|}{ General Motor Parameters } & \multicolumn{2}{|c|}{ Development Kit Information } \\
\hline$\check{\tau}$ & $0.01 \mathrm{Nm}$ & DSC & MC56F84789 \\
\hline$\check{p}$ & $40 \mathrm{~W}$ & MIPS & 100 \\
\hline$\check{\omega}$ & $4000 \mathrm{rpm}$ & $\mathrm{AD}$ & 12 bits \\
\hline
\end{tabular}

Experimental Set Parameters

- For the delays, a delay model was added, obtained with Padè's approximation.

- An anti wind-up logic was used in PIs implementation, since virtual saturation limits are included.

- By using frequency response method, two conservative PIs were designed (PI01 and PI02). The first was designed for phase margin (PM) of $40^{\circ}$ and cutoff frequency $f_{c}$ of $6 \mathrm{~Hz}$. The other was designed for $\mathrm{PM}$ of $60^{\circ}$ and $f_{c}=3 \mathrm{~Hz}$ (plant original pole is about $4 \mathrm{~Hz}$ ).

- Also, by using the same method, two aggressive PIs were designed (PI03 - with $\mathrm{PM}=60^{\circ}$ and $f_{c}=$ $19 \mathrm{~Hz}-$ and PI04 - with $\mathrm{PM}=70^{\circ}$ and $f_{c}=40 \mathrm{~Hz}$ ).

- Other six PIs were tested. Their gains were obtained by varying the gains of PIs used before (the complete information about them is presented in Table I).

- Finally, the PI controllers were discretized using Euler's backward discretization. All PIs tested with $f_{c}>80 \mathrm{~Hz}$ or $\mathrm{PM}<15^{\circ}$ caused instability problems.

Figure 3 presents the proposed predictive control scheme, with the internal structure of SSMPC. To observe the effects of proposed model and used cost function, the following procedure was adopted:

- Observing the effect of delays treatment. 
TABLE III

Comparison of SSMPC and PI Controllers

\begin{tabular}{lccccc}
\hline Controller & $q_{e}^{\diamond}$ & $q_{u}^{\square}$ & $m_{p}^{*}$ & $t_{r}^{\dagger}$ & $t_{s}^{\ddagger}$ \\
\hline MPC 01 & 2671 & 0.130466 & $15.0 \%$ & 56.0 & 110 \\
\hline MPC 02 & 417 & 0.137979 & $3.10 \%$ & 48.0 & 90 \\
\hline PI 01 & 4609 & 0.130094 & $21.3 \%$ & 57.6 & 125 \\
\hline PI 02 & 7456 & 0.117501 & $3.20 \%$ & 99.0 & 120 \\
\hline PI 03 & 321 & 0.139789 & $6.50 \%$ & 49.1 & 92 \\
\hline PI 04 & 420 & 0.143715 & $5.11 \%$ & 46.5 & 89 \\
\hline PI 05 & 15135 & 0.150644 & $28.0 \%$ & 44.2 & $>1000$ \\
\hline PI 06 & 7032 & 0.182382 & $5.22 \%$ & 46.1 & $>1000$ \\
\hline PI 07 & 7370 & 0.118118 & $3.40 \%$ & 99.5 & 157 \\
\hline PI 08 & 4484 & 0.130098 & $20.8 \%$ & 58.3 & 300 \\
\hline PI 09 & 4903 & 0.132939 & $24.4 \%$ & 53.6 & 579 \\
\hline PI 10 & 11786 & 0.109718 & $0.0 \%$ & 535 & 555 \\
\hline
\end{tabular}

Average quadratic error.

$\square$ Root-mean-squared duty cycle in all cycle.

${ }^{*}$ Maximum peak (step of $700 \mathrm{rpm}$, when $y_{r}=400 \mathrm{rpm}$ )

${ }^{\dagger}$ Rise time (ms) from $0 \%$ to $90 \%$ (step of $700 \mathrm{rpm}$, when $y_{r}=400 \mathrm{rpm}$ ).

${ }^{\ddagger}$ Settling time (ms), $2 \%$ criteria (step of $700 \mathrm{rpm}$, when $y_{r}=400 \mathrm{rpm}$ ).

- Testing a conservative SSMPC tuning (MPC01) and an aggressive SSMPC tuning (MPC02), varying $\rho$ parameter, which is responsible for penalizing energy flow in the system (MPC01 has $\rho=15000$ and MPC02 has $\rho=750$ ). Both horizons were maintained constant at two steps ahead of modeled delay.

Table II presents the parameters of the experimental setup. In this table, $f_{s}$ is the switching frequency, $\breve{\tau}$, $\check{p}$ and $\breve{\omega}$ are, respectively, rated torque, power and mechanical speed.

\section{RESULTS AND DISCUSSION}

The performance comparison between the MPCs and the PIs is presented in Table III. Figure 5 exhibits transient speed performance of PI03, PI04 and MPC02. Figure 6 exhibits transient duty cycle for same period evaluated in Figure 5. Analyzing Figure 5, MPC start the trajectory segment before PIs due to delay treatment, since it was not used the anticipated reference resource of SSMPC (see [16] for more information). Both PIs have greater speed and duty cycle peaks than SSMPC. It shows that predictive control can have the similar dynamic performances that PIs but with lower

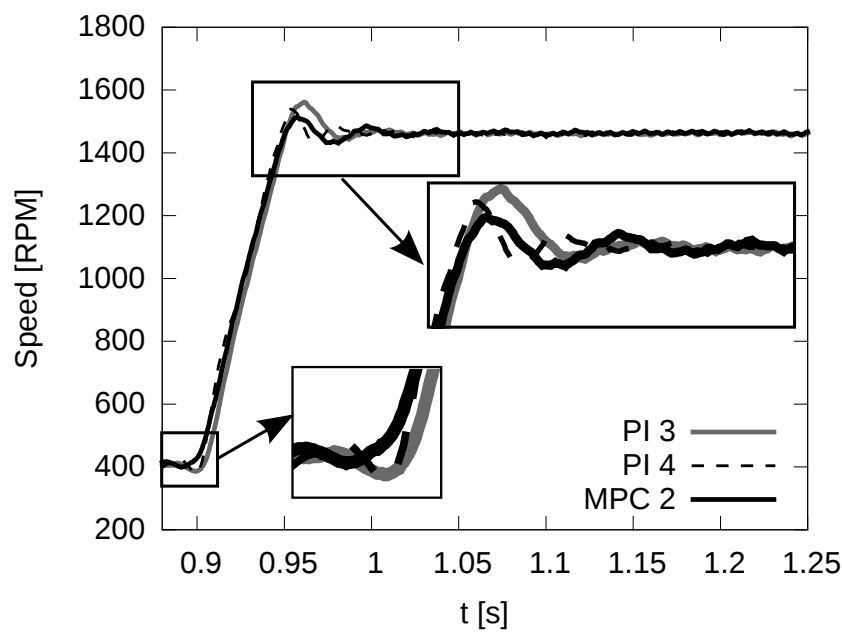

Fig. 5. Comparison of speed dynamics among PI03, PI04 and MPC02.
TABLE IV

Computational Cost Analysis

\begin{tabular}{lcc}
\hline Controller & Average processing time & Percent in relation to $t_{s}{ }^{1}$ \\
\hline PI (float) & $141 \mu \mathrm{s}$ & $14.1 \%$ \\
\hline SSMPC $^{2}($ float $)$ & $185 \mu \mathrm{s}$ & $18.5 \%$ \\
\hline PI $\left(\right.$ Frac16 $\left.^{3}\right)$ & $0.09 \mu \mathrm{s}$ & $0.09 \%$ \\
\hline SSMPC $($ Frac16) & $0.36 \mu \mathrm{s}$ & $0.36 \%$ \\
\hline
\end{tabular}

${ }^{1}$ The considered sampling time is $1.0 \mathrm{~ms}$. This sampling time is high because PMW needs to perform voltage average value and there are SPI communication between switches pre-driver MC33937 and the digital controller, that takes approximately $200 \mu \mathrm{s}$.

${ }^{2}$ For $h_{p}=h_{c}=5$.

${ }^{3}$ This is a special data type from Freescale DSC, with range [-1.0,0.9999]. It is a fixed point type with special arithmetic instructions.

peaks and spent energy due to the optimization process. In Table III, it is possible to observe that MPC02 spent lower energy than both PIs and have lower a average quadratic error than PI04. Except by PI 10, which is over damped, MPC02 has the lowest speed peak and the second lowest settling time.

Figure 7 presents the transient speed performance of PI01, PI02 and MPC01. Figure 8 shows transient duty cycle of same period evaluated in Figure 5. These controllers have conservative characteristics if compared with the previous. Since their cut-off frequency is lower, the controllers take more time to act on the speed error. It is possible to note that MPC01 is almost in the same frequency than PI01, but it has lower peaks in speed tracking and duty cycle. Due to this, SSMPC converges faster than both PIs in $2 \%$ criteria. In Table III, it can be observed that MPC01 has a lower $q_{e}$ than any other controller with similar $q_{u}$ or $t_{r}$, showing benefits of optimization process. Other interesting fact is that in steady state, the controllers analyzed in Figure 8 spent less energy than the previous analyzed in Figure 6, since they have lower duty cycle oscillation, and, this way, they do not act over drive noise.

Figures 9 and 10 show, respectively, MPC02 in speed performance and duty cycle for full proposed tracking routine. It can be observed that MPC was able to maintain its performance over different operating points.

Table IV presents the results for computational cost analysis. All costs respect the controller sampling time.

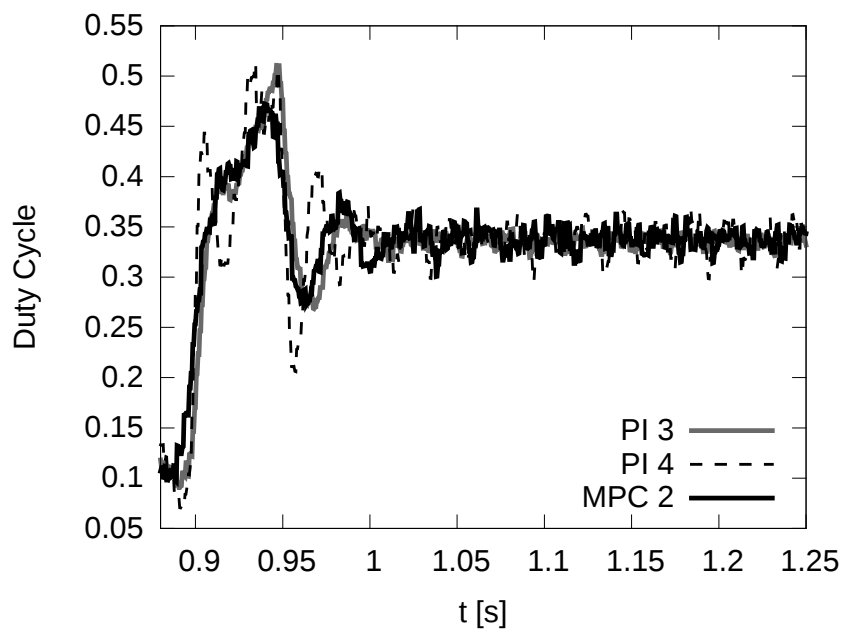

Fig. 6. Duty cycle comparison of MPC02, PI03 and PI04. 


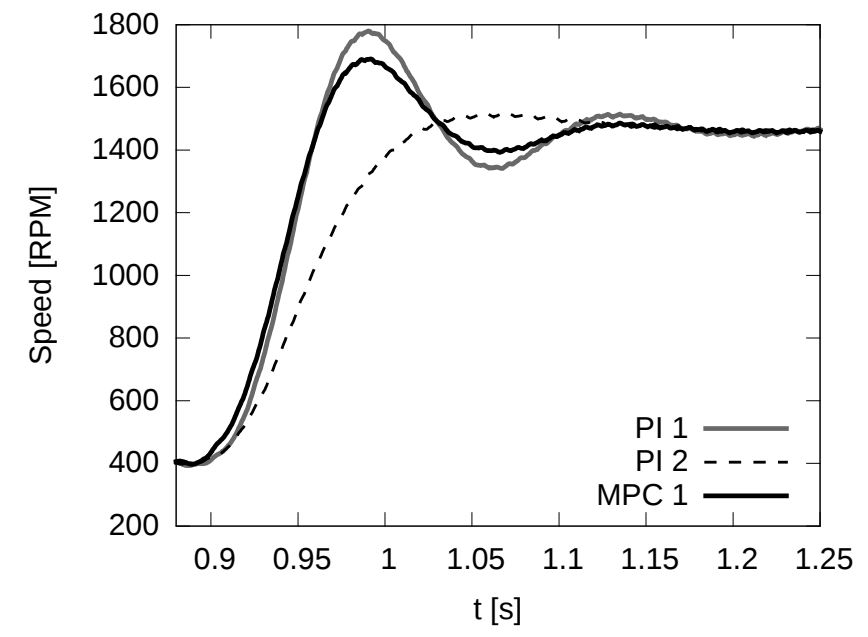

Fig. 7. Speed performance of conservative PIs and MPC01.

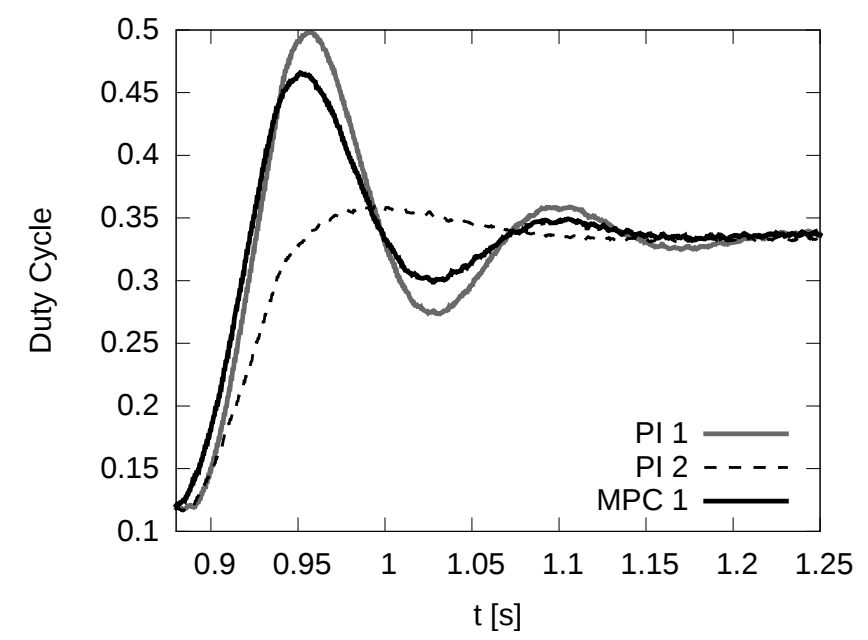

Fig. 8. Duty cycle comparison of conservative PIs and MPC01.

However, the values fill about $15-25 \%$ of sampling time. This large values occurs due to the float data type used in the control strategies programming. The DSC only emulates floating point, at a higher processing cost. Using fixed point variables is more computationally efficient. This can be done with the 16 bit data type from Freescale called Frac16 with range [-1,0.9999]. With this data type, the controllers are much more faster than using floating point, but it has the disadvantage of fixed point programming.

\section{CONCLUSION}

This paper presented a predictive control scheme for speed control of a PMSM. The drive dynamics using the proposed control approach was compared with PI controllers. Two comparative approaches are followed: dynamic performance and energy cost. For similar dynamic performance, SSMPC have lower energy cost. For similar energy cost, SSMPC have better dynamic performance. It is really easy to tune predictive controllers since just one parameter is responsible to energy penalization. It demonstrates the benefits of optimization process within MPC. Predictive controllers also treat drive delays in the model, starting trajectory tracking faster. Still, a computational cost analysis was done. All controllers

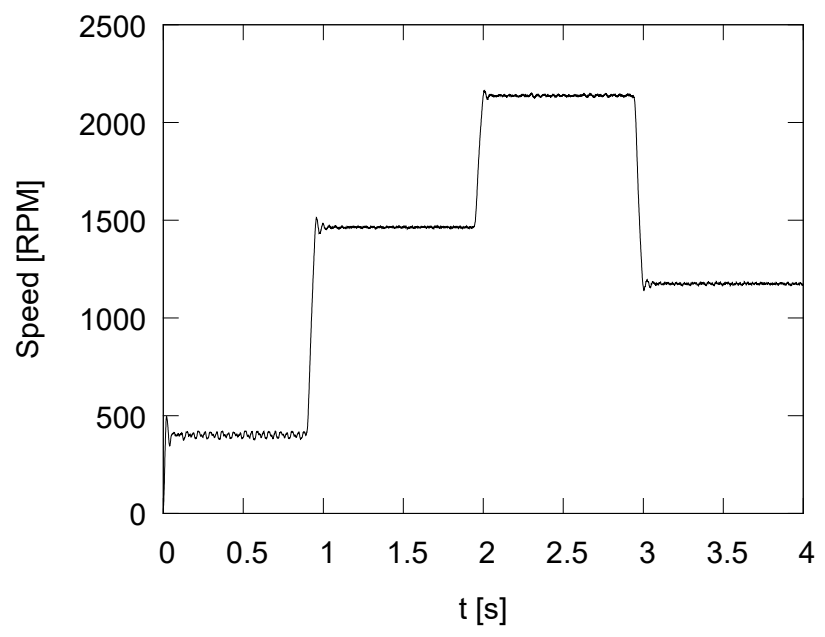

Fig. 9. Complete speed tracking routine for MPC02.

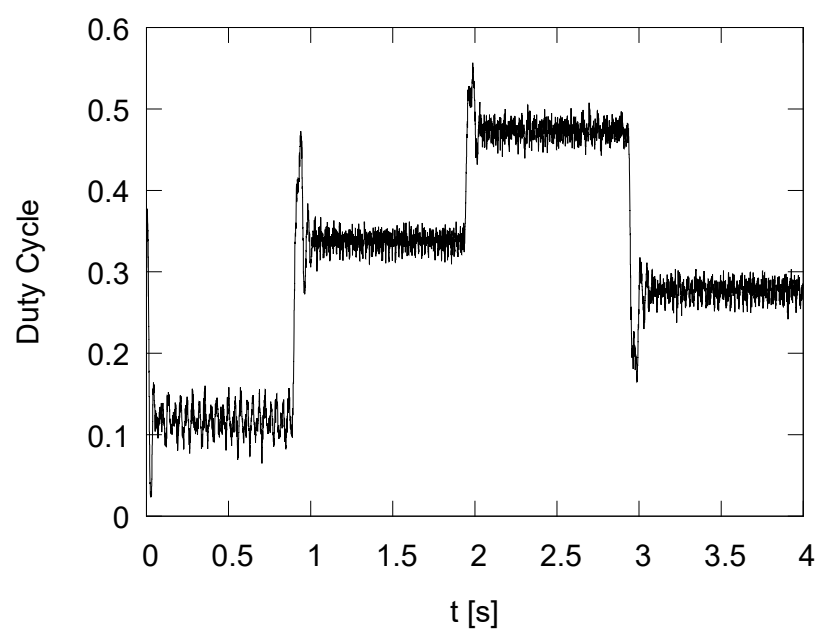

Fig. 10. Duty cycle for complete speed tracking routine for MPC02.

have similar processing time, but they are high due to the use of floating point. By using fixex point variables, the computational cost has a significant reduction.

For future works, a multi-model approach will be proposed, to achieve better results in different operating regions and to perform speed and current control of PMSMs, using more complex predictive techniques.

\section{ACKNOWLEDGEMENT}

The authors thank CNPq and CAPES for financial support. Also, the authors thank Freescale Semiconductors for TWR56F84000 development kit donation.

\section{REFERENCES}

[1] M. A. Rahman, "History of Interior Permanent Magnet Motors", IEEE Industrial Applications Magazine, vol. 19, no. 1, pp. 10-15, Jan/Feb 2013.

[2] W. Zhao, M. Cheng, K. Chau, C. Chan, "Control and operation of fault-tolerant flux-switching permanentmagnet motor drive with second harmonic current injection", IET Electric Power Applications, vol. 6, no. 9, pp. $707-715$, Nov 2012. 
[3] M. Miyamasu, K. Akatsu, "Efficiency comparison between Brushless dc motor and Brushless AC motor considering driving method and machine design", in 37th Annual Conference on IEEE Industrial Electronics Society (IECON), pp. 1830 - 1835, 2011.

[4] K. Tabarraee, J. Iyer, J. Jatskevich, "Average-value modeling of brushless DC motors With trapezoidal back-EMF", in 11th IEEE International Symposium on Industrial Electronics (ISIE), pp. 531 - 537, 2011.

[5] K. Chau, C. Chan, C. Liu, "Overview of PermanentMagnet Brushless Drives for Electric and Hybrid Electric Vehicles", IEEE Transactions on Industrial Electronics, vol. 55, no. 6, pp. 2246-2257, june 2007.

[6] C. L. Baratieri, H. Pinheiro, "I-f Starting Method For Smooth And Fast Transition To Sensorless Field Oriented Control Of Bldc Motors", Eletrônica de Potência, vol. 19, no. 3, pp. 268 - 276, Aug 2014.

[7] M. Bevilaqua, A. Nied, J. de Oliveira, "Labview FPGA FOC implementation for synchronous Permanent Magnet Motor Speed Control", in 11th IEEE/IAS International Conference on Industry Applications (INDUSCON), pp. 1-8, 2014.

[8] E. M. Fernandes, A. C. Oliveira, A. M. N. Lima, C. B. ao Jacobina, "Estimação da posição rotórica do motor PMSM com minimização da distorção de tensão de alta frequência”, Eletrônica de Potência, vol. 17, no. 1, pp. 447-435, Feb 2012.

[9] G. S. Buja, M. P. Kazmierkowski, "Direct Torque Control of PWM Inverter-Fed AC Motors - A Survey", IEEE Transactions on Industrial Electronics, vol. 51, no. 4, pp. 744-757, Aug 2004.

[10] A. Gaeta, G. Scelba, A. Consoli, "Sensorless Vector Control of PM Synchronous Motors During Single-Phase Open-Circuit Faulted Conditions", IEEE Transactions on Industry Applications, vol. 48, no. 6, pp. 1968-1979, Dec 2012.

[11] P. Pillay, R. Krishnan, "Modeling of permanent magnet motor drives", IEEE Transactions on Industrial Electronics, vol. 35, no. 4, pp. 537-541, Nov 1988.

[12] S. Ozturk, W. Alexander, H. Toliyat, "Direct Torque Control of Four-Switch Brushless DC Motor With Non-Sinusoidal Back EMF", IEEE Transactions on Power Electronics, vol. 25, no. 2, pp. 263 - 271, Feb 2010.

[13] G. Negri, A. Bartsch, M. Cavalca, J. de Oliveira, A. Nied, A. Silveira, "Model-based predictive direct speed control applied to a Permanent Magnet Synchronous Motor with trapezoidal back-EMF", in 11th IEEE/IAS International Conference on Industry Applications (INDUSCON), pp. 1-8, 2014.

[14] S. J. Qin, T. A. Badgwell, "A survey of industrial model predictive control technology", Control Engineering Practice, vol. 11, no. 7, pp. 733-764, July 2003.

[15] A. G. Bartsch, A. Nied, M. S. M. Cavalca, J. de Oliveira, "Evaluation of Constrained and Unconstrained SESSMPC Applied in Five-Phase PMSM", in 24th International Symposium on Industrial Electronics (ISIE), pp. 494 - 499, 2015.
[16] A. G. Bartsch, M. S. M. Cavalca, A. Nied, J. de Oliveira, "Evaluation of Constrained SESSMPC to Drive a Three-Phase PMSM Applied in Washing Machines", in 24th International Symposium on Industrial Electronics (ISIE), pp. 500 - 505, 2015.

[17] E. de Santana, E. Bim, W. do Amaral, "A Predictive Algorithm for Controlling Speed and Rotor Flux of Induction Motor", IEEE Transactions on Industrial Electronics, vol. 12, no. 12, pp. 4398 - 4407, Dec 2008.

[18] M. Preindl, S. Bolognani, "Model Predictive Direct Torque Control With Finite Control Set for PMSM Drive Systems, Part 2: Field Weakening Operation", IEEE Transactions on Industrial Informatics, vol. 9, no. 2, pp. 648-657, Apr 2013.

[19] T. Geyer, G. Papafotiou, M. Morari, "Model Predictive Direct Torque Control-Part I: Concept, Algorithm, and Analysis", IEEE Transactions on Industrial Electronics, vol. 56, no. 6, pp. 1894-1905, June 2009.

[20] M. Cavalca, R. Galvaao, T. Yoneyama, "Integrator resetting with guaranteed feasibility for an LMI-based robust model predictive control approach", in 18th Mediterranean Conference on Control \& Automation (MED), pp. 634 - 639, 2010.

[21] R. L. Valle, A. A. Ferreira, J. G. de Oliveira, V. F. Montagner, P. G. Barbosa, "Projeto e implementação de um controlador digital preditivo para regular as correntes de um motor BLDC", Eletrônica de Potência, vol. 20, no. 2, pp. 215-224, May 2015.

[22] Yokogawa, DL850E/DL850EV ScopeCorder Getting Started Guide, 2013.

\section{BIOGRAPHIES}

Arthur G. Bartsch, born in 10/20/1992 in Joinville-SC, is an Electrical Engineer (2015), and he is currently working toward the Master degree at the State University of Santa Catarina, Joinville, Brazil. His areas of interest are power electronics, drives and predictive control.

Gabriel H. Negri, born in 03/12/1992 in Joinville-SC, is an Electrical Engineer (2015), and he is currently working toward the Master degree at the State University of Santa Catarina, Joinville, Brazil. His areas of interest are nonlinear control and optimization.

Camila R. Scalabrin, born in 04/02/1994 in Sananduva-RS, is an Electrical Engineering student at the State University of Santa Catarina, Joinville, Brazil. Her areas of interest are electrical machines and drives.

Mariana S. M. Cavalca, born in 06/05/1984 in São José dos Campos-SP, is a Control and Automation Engineer (2007), Master (2008) and Doctor in Electronic and Computing Engineering (2011) with Technological Institute of Aeronautics. Between 2012 and 2015 she was coordinator of Industrial Automation Laboratory. She is currently an Assistant Professor with the Department of Electrical Engineering, State University of Santa Catarina, Joinville, 
Brazil. Her areas of interest are predictive control, faulttolerant control and optimal control.

Ademir Nied, born in 06/12/1962 in Santo Ângelo-RS, is an Electrical Engineer (1987), Master (1995) and Doctor in Electrical Engineering (2007) with Federal University of Minas Gerais. He is currently an associate professor with the Department of Electrical Engineering, State University of Santa Catarina, Joinville, Brazil. His areas of interest are electrical machines, control of electrical drives and neural networks.

José de Oliveira, José de Oliveira, born in 06/15/1961 in Mandaguari-PR, is an Electrical Engineer (1986), Master (1994) and Doctor in Electrical Engineering (1990) with the Federal University of Santa Catarina. He is currently an associate professor with the Department of Electrical Engineering, State University of Santa Catarina, Joinville, Brazil. His areas of interest are control systems, electrical machine actuation and power electronics. 